\title{
On tension-torsion testing of solid cylindrical specimens
}

\author{
R.M. Kashaev ${ }^{\dagger}$ \\ †rishat@kashaev.ru \\ Institute for Metals Superplasticity Problems of RAS, 39 Khalturin str., Ufa, 450001, Russia
}

One of the features of the rheological behavior of metals and alloys at high temperatures is their high strain rate sensitivity. Here, a method is proposed to study the relationship between stresses and strain rates basing on the tests of cylindrical specimens deformed in tension-torsion under non-proportional deformation (complex loading). A strain path in the form of a two-segment broken line is considered. The deformation process is specified by two components of the strain rate tensor (axial and shear ones) as functions of time. At every instant, the axial force and torque corresponding to the functions are recorded. A relationship between the force parameters (axial force and torque) recorded in the experiments, the specified kinematic parameters (the rates of tension and torsion), the strain rate sensitivity coefficient, the strain hardening exponent and the approach angle between the vectors of stress and the strain rate on the surface of the specimen under non-proportional deformation is obtained. Particular cases of deformation paths of the two-segment broken line type, where the second segment is a uniaxial tension, pure torsion or combined tension -torsion, are considered. Analytic formulas to calculate the stress vector and its components, as well as the approach angle characterizing the direction of the stress vector in relation to the strain rate vector, are derived for the points on the specimen's surface. It is shown that the method proposed is a generalization of the experimental technique of plotting shear diagrams according to the torsion test data for a strain rate and strain sensitive material based on the Fields-Backofen equation. The method was used to process the experimental data obtained in tensiontorsion testing of the Ti-6 Al-4V titanium alloy under non-proportional deformation under conditions of superplasticity. When processing the test data, the compliance of the "specimen - testing machine" system was taken into account.

Keywords: complex loading, superplasticity.

УДК: 539.3

\section{Об испытании сплошных цилиндрических образцов при растяжении с кручением}

\author{
Кашаев Р. М. ${ }^{\dagger}$ \\ Институт проблем сверхпластичности металлов РАН, ул.Халтурина 39, Уфа, 450001, Россия
}

Особенностью реологического поведения металлов и сплавов при высокой температуре является их повышенная чувствительность к скорости деформирования. Предлагается метод исследования связи напряжений со скоростями деформаций на основании испытаний сплошных цилиндрических образцов, деформируемых растяжением с кручением в условиях непропорционального деформирования. Рассматривается плоская траектория деформаций вида двухзвенной ломаной. Процесс деформирования задается двумя компонентами тензора скоростей деформаций (осевой и сдвиговой) в виде функций времени. В каждый момент времени регистрируются соответствующие им осевое усилие и крутящий момент. Получено уравнение связи регистрируемых в эксперименте силовых параметров (осевого усилия и крутящего момента), заданных кинематических параметров (скорости растяжения и скорости кручения), коэффициентов деформационного и скоростного упрочнения материала и угла сближения между векторами напряжения и скорости деформаций на поверхности образца при непропорциональном деформировании. Рассмотрены частные случаи траекторий деформаций вида двухзвенной ломанной, когда второе звено является одноосным растяжением, чистым кручением или растяжением с одновременным кручением. Для точек, расположенных на цилиндрической поверхности образца приведены формулы для вычисления вектора напряжений и его компонентов, а также угла сближения (ориентации вектора напряжений по отношению к вектору скоростей деформаций) из экспериментальных зависимостей осевого усилия и крутящего момента от времени при заданных значениях скоростей растяжения и кручения. Показано, что предлагаемая методика является обобщением экспериментальной методики построения диаграммы сдвига по данным испытаний на кручение материала, чувствительного к скорости и степени деформации, в основе которой лежит формула Филдса-Бэкофена. Предлагаемая методика была использована при обработке экспериментальных данных, полученных при испытании титанового сплава Ti-6Al-4V 
при непропорциональном деформировании в условиях сверхпластичности. При обработке данных учитывалась податливость системы «образец - испытательная машина».

Ключевые слова: сложное нагружение, сверхпластичность.

В теории упругопластических процессов вводится понятие о векторных свойствах материала [1-5]. Процесс деформирования в некоторой фиксированной точке среды задается тензором деформации $e_{i j}(t)$. Ему ставится в соответствие вектор деформаций е $(t)$, годограф которого называется траекторией деформаций. При экспериментальном исследовании векторных свойств изучается отклонение вектора напряжений $\boldsymbol{\sigma}(t)$ от касательной к траектории деформаций. Традиционными при исследовании непропорционального деформирования (сложного нагружения) являются плоские траектории деформаций, реализуемые в опытах на растяжение с кручением. Как правило, исследуются ломаные траектории, состоящие из отдельных прямолинейных отрезков. Простейшей ломаной траекторией является двухзвенная ломаная, состоящая из двух отрезков, ориентированных под некоторым углом $\vartheta_{0}$ друг к другу. Основные параметры двухзвенной траектории деформаций указаны на Рис. 1 . В частности, $\vartheta_{0}-$ угол излома траектории деформации (если второе звено траектории отклоняется от направления первого звена по ходу часовой стрелки вправо, то $\vartheta_{0}<0$, если против часовой стрелки, то $\left.\vartheta_{0}>0\right) ; S_{0}$ - длина траектории деформации в первом звене в точке излома $A$; $\vartheta-$ угол сближения на длине второго звена $\Delta S$, отсчитываемой от точки излома траектории деформации; $h-$ след запаздывания векторных свойств (длина второго звена до точки B), по исчерпанию которой вектор напряжений $\boldsymbol{\sigma}$ становится коллинеарен (как правило с некоторым допуском) вектору скорости деформации $\boldsymbol{\xi}$.

Особенностью высокотемпературного деформирования металлов является их чувствительность к скорости деформации. Влияние скорости деформирования на механическое поведение металлов при высоких температурах исследовано, в основном в условиях пропорционального (простого), прежде всего одноосного, деформирования. Известны экспериментальные работы

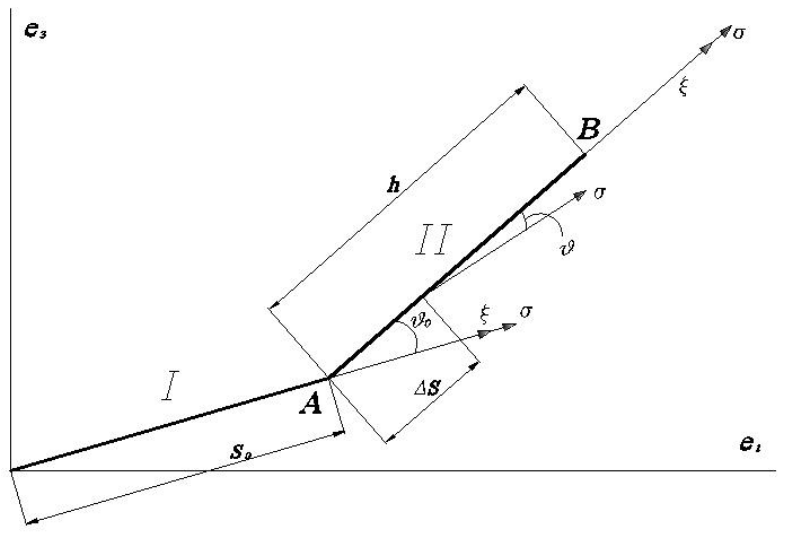

Pис. 1. Траектория деформации в плоскости $\left(e_{1}, e_{3}\right)$.

Fig. 1. Strain trajectory in $\left(e_{1}, e_{3}\right)$ plane на сложное нагружение полиэтилена высокого давления, материала чувствительного к скорости деформации $[6,7]$. В работе [8] проведен анализ влияния скорости деформации на законы изменения модуля и угла сближения вектора напряжений в процессах сдвига эвтектики олова и свинца по результатам экспериментальных данных по кручению [9].

В процессе экспериментов на непропорциональное деформирование (сложное нагружение), как правило, используются тонкостенные трубчатые образцы [10 - 13]. Поскольку толщина образца по сравнению с его радиусом достаточно мала, неоднородностью распределения напряжений и деформаций по толщине образца пренебрегают, что и делает возможным их определение по экспериментальным данным. Однако этот метод имеет существенные недостатки: невозможность (вследствие потери устойчивости) получения больших деформаций; трудоемкость изготовления образцов; большая относительная погрешность регистрации силовых параметров ввиду их низкого значения при высокотемпературных испытаниях. Более предпочтительным является метод использования сплошных цилиндрических образцов $[14,15]$. Однако при этом ввиду неоднородного напряженно-деформированного состояния в образцах возникает проблема расшифровки результатов экспериментов. Поэтому, возникает необходимость внесения некоторых допущений для полного восстановления распределения напряжений. Наибольшая неоднородность напряжений, деформаций и скоростей деформаций в поперечном сечении образца возникает при чистом кручении. При высокотемпературных исследованиях на кручение напряжения сдвига чаще всего рассчитывают по формуле Филдса-Бэкофена [16]:

$$
\tau(R)=M(3+m+n) / 2 \pi R^{3},
$$

где $\tau(R)$ - напряжение сдвига на поверхности образца; $M$ - крутящий момент; $R$ - радиус образца; $m$ и $n-$ соответственно коэффициенты скоростного и деформационного упрочнения (постоянные материала). Коэффициенты $m$ и $n$ в формуле (1) учитывают неравномерность скорости и степени деформации по сечению образца при кручении. Коэффициент $m$ может быть определен, например по двум измерениям крутящих моментов $M_{1}$ и $M_{2}$ при соответствующих им разных скоростях кручения $\omega_{1}$ и $\omega_{2}$ :

$$
m=\frac{\ln \left(M_{1} / M_{2}\right)}{\ln \left(\omega_{1} / \omega_{2}\right)} \text { при } \varphi_{1}=\varphi_{2} \text { и } \omega_{1} \neq \omega_{2},
$$

а коэффициент деформационного упрочнения $n$ :

$$
n=\frac{\ln \left(M_{1} / M_{2}\right)}{\ln \left(\varphi_{1} / \varphi_{2}\right)} \text { при } \omega=\text { const и } \varphi_{1} \neq \varphi_{2},
$$

где $\varphi_{1}$ и $\varphi_{2}-$ углы закручивания, соответствующие $M_{1}$ и $M_{2}$. 
Для вывода формулы (1) была использована степенная зависимость напряжения сдвига $\tau$ от степени и скорости деформаций в поперечном сечении образца:

$$
\tau=K \cdot \dot{\gamma}^{m} \gamma^{n},
$$

где $\gamma=\varphi \cdot r / L$ и $\dot{\gamma}=\omega \cdot r / L-$ соответственно деформация и скорость сдвига в точках образца, расположенных на расстоянии $r$ от его оси; $L-$ длина расчетной части образца; $K, m, n-$ постоянные материала. Эта модель для одноосного растяжения имеет вид [17]:

$$
\sigma=K \xi^{m} p^{n},
$$

где $\sigma-$ напряжение; $\xi-$ скорость деформации; $p=\int_{0}^{T} \xi(t) \mathrm{d} t-$ параметр Одквиста (истинная деформация).

Для пересчета полученных при кручении значений $\tau(R), \gamma(R)$ и $\dot{\gamma}(R)$ на поверхности образца в соответствующие значения интенсивностей напряжений $\sigma_{e}(R)$, деформаций $e(R)$, и скоростей деформаций $\xi_{e}(R)$ используют следующие формулы (по Мизесу) [16]:

$$
\begin{gathered}
\sigma_{e}(R)=\sqrt{3} \tau(R)=\frac{\sqrt{3} M(3+m+n)}{2 \pi R^{3}}, \\
e(R)=\frac{\gamma}{\sqrt{3}}=\frac{\varphi R}{\sqrt{3} L}, \quad \xi_{e}(R)=\frac{\dot{\gamma}}{\sqrt{3}}=\frac{\omega R}{\sqrt{3} L} .
\end{gathered}
$$

При одноосном же растяжении, ввиду однородности напряженно-деформированного состояния, напряжения вычисляют по стандартной формуле:

$$
\sigma_{e}=P / \pi R^{2},
$$

где $P$ - осевое усилие, $R$ - текущий радиус образца.

Рассмотрим пример №1 (общий случай). Сплошной цилиндр (стержень) с начальным радиусом $R_{0}$ и начальной длиной $L_{0}$ нагружается по кинематической программе растяжением с одновременным кручением, состоящей из двух звеньев, которые отличаются соотношением сдвиговой и осевой составляющих скорости деформации. Полагаем, что при деформировании материал цилиндра ведет себя как изотропная среда и для напряжений течения предполагается справедливой гипотеза «единой кривой», связывающее интенсивности напряжений, деформаций и скоростей деформаций [18]. При этом векторные свойства материала не зависят от скорости деформации, даже при скачкообразном ее изменении $[6,7]$. В процессе испытания в каждый момент времени $t$ регистрируем абсолютное удлинение $\Delta L(t)$, угол закручивания $\varphi(t)$, осевую силу $P(t)$ и крутящий момент $M(t)$. Текущую длину цилиндра $L(t)$ вычисляем по формуле: $L(t)=L_{0}+\Delta L(t)$, а текущий радиус $R(t)$ вычисляем из условия объемной несжимаемости материала по формуле: $R(t)=R_{0} \sqrt{L_{0} / L(t)}$. Будем считать, что напряженно-деформированное состояние не изменяется вдоль оси цилиндра. При этом ввиду осевой симметрии компоненты тензоров напряжений, деформаций и скоростей деформаций в цилиндрической системе координат $(r, \varphi, z)$ (ось $O z$ совпадает с осью цилиндра, ось $O r$ направлена вдоль радиуса) являются функциями только координаты $r$. Далее предполагаем, что при деформировании цилиндра выполняются гипотезы пло- ских сечений и прямолинейности радиальных волокон, обычно принимаемые в таких случаях. Деформации считаем малыми. При выполнении условия объемной несжимаемости материала, для компонент тензоров деформаций $e_{i j}(r, t)$ и скоростей деформаций $\xi_{i j}(r, t)$ имеем следующие выражения [17]:

$$
\begin{aligned}
& e_{r r}=e_{\varphi \varphi}=-\Delta L / 2 L, e_{z z}=\Delta L / L, e_{r \varphi}=e_{r z}=0, e_{\varphi z}=\varphi r / 2 L, \\
& \xi_{r r}=\xi_{\varphi \varphi}=-V / 2 L, \quad \xi_{z z}=V / L, \quad \xi_{r \varphi}=\xi_{r z}=0, \quad \xi_{\varphi z}=\omega r / 2 L,
\end{aligned}
$$

где $V$ - осевая скорость деформирования, $\omega-$ угловая скорость кручения.

Тензорам напряжений $\sigma_{i j}(r, t)$, деформаций $e_{i j}(r, t)$ и скоростей деформаций $\xi_{i j}(r, t)$ в точках цилиндра, находящихся на расстоянии $r$ от его оси, поставим в соответствие векторы напряжений $\boldsymbol{\sigma}(t)$, деформаций $\mathbf{e}(t)$ и скоростей деформаций $\xi(t)$, для компонент которых, исходя из $[5,10,15]$ приняты следующие выражения:

$$
\begin{gathered}
\sigma_{1}=\sigma_{z z^{\prime},}, \sigma_{3}=\sqrt{3} \sigma_{\varphi z^{2}}, \sigma_{2}=\sigma_{4}=\sigma_{5}=0, \\
e_{1}=\Delta L / L, \quad e_{3}=\varphi r / \sqrt{3} L, \quad e_{2}=e_{4}=e_{5}=0, \\
\xi_{1}=V / L, \quad \xi_{3}=\omega r / \sqrt{3} L, \quad \xi_{2}=\xi_{4}=\xi_{5}=0,
\end{gathered}
$$

Из соотношений (6) следует, что для всех точек цилиндра, траектории деформаций, соответствующие процессу растяжения с одновременным кручением, являются двумерными и лежат в плоскости $\left(e_{1}, e_{3}\right)$, а модули векторов $\boldsymbol{\sigma}, \mathbf{e}, \boldsymbol{\xi}$ равны соответствующим интенсивностям напряжений $\sigma_{e}$, деформаций $e_{e}$ и скоростей деформаций $\xi_{e}$ и имеют следующий вид:

$$
\begin{gathered}
\sigma_{e}=\sqrt{\sigma_{z z}^{2}+3 \sigma_{\varphi z}^{2}}, \\
e_{e}=\Delta L / L \sqrt{q^{2} \rho^{2}+1}, \\
\sigma_{e}=V / L \sqrt{q^{2} \rho^{2}+1},
\end{gathered}
$$

где $\rho=r / R-$ относительный радиус цилиндра, $q=\omega R / \sqrt{3} V-$ безразмерный параметр, характеризующий закон деформирования и представляющий собой соотношение ненулевых компонент вектора скоростей деформации (6) для точек цилиндра, находящихся на его боковой поверхности.

Из условия равновесия, учитывая (6), для осевой силы $P$ и крутящего момента $M$ имеем следующие выражения [15]:

$$
\begin{gathered}
P=2 \pi \int_{0}^{R} \sigma_{z z} r \mathrm{~d} r=2 \pi R^{2} \int_{0}^{1} \sigma_{1} \rho \mathrm{d} \rho, \\
M=2 \pi \int_{0}^{R} \sigma_{\varphi z} r^{2} \mathrm{~d} r=\left(2 \pi R^{3} / \sqrt{3}\right) \int_{0}^{1} \sigma_{3} \rho^{3} \mathrm{~d} \rho .
\end{gathered}
$$

Выражаем скалярное произведение векторов напряжений и скоростей деформаций через их компоненты:

$$
\sigma_{e} \xi_{e} \cos \vartheta=\sigma_{1} \xi_{1}+\sigma_{3} \xi_{3},
$$

где $9=9(R) \rho$ - угол между векторами напряжений и скорости деформаций (угол сближения) в точках, находящихся на расстоянии $r$ от его оси, $\vartheta(R)$ - угол сближения в точках цилиндра, находящихся на его боковой поверхности, т. е. при $\rho=r / R=1$.

Решая совместно (9) и уравнение для $\sigma_{e}$ из (7) получим выражения для компонент вектора напряжений:

$$
\begin{aligned}
& \sigma_{1}=\left(\xi_{1} \cos \vartheta+\xi_{3} \sin \vartheta\right) \sigma_{e} / \xi_{e}, \\
& \sigma_{3}=\left(\xi_{3} \cos \vartheta-\xi_{1} \sin \vartheta\right) \sigma_{e} / \xi_{e},
\end{aligned}
$$


Используя (2) и принимая во внимание (7) связь между интенсивностью напряжений, с одной стороны, и интенсивностями деформации и скоростей деформаций, с другой в каждой точке образца представим в виде:

$$
\begin{aligned}
\sigma_{e}=\sigma_{e}(R) & {\left[\xi_{\mathrm{e}} / \xi_{\mathrm{e}}(R)\right]^{m}\left[e_{e} / e_{e}(R)\right]^{n}=} \\
= & \sigma_{e}(R) \sqrt{\left[\left(q^{2} \rho^{2}+1\right) /\left(q^{2}+1\right)\right]^{m+n}},
\end{aligned}
$$

где $\sigma_{e}(R), \xi_{e}(R), e_{e}(R)-$ соответственно интенсивности напряжений, скоростей деформаций и деформации на поверхности образца.

Подставляя (11) соответственно в (10) получим закон распределения компонент вектора напряжений по поперечному сечению сплошного цилиндра:

$$
\begin{aligned}
& \sigma_{1}=\sigma_{e}(R)\{\cos [\vartheta(R) \rho]+q \rho \sin [\vartheta(R) \rho]\} \cdot \\
& \cdot \sqrt{\left(q^{2} \rho^{2}+1\right)^{m+n-1} /\left(q^{2}+1\right)^{m+n}}, \\
& \sigma_{3}=\sigma_{e}(R)\{q \rho \cos [\vartheta(R) \rho]-\sin [\vartheta(R) \rho]\} \cdot \\
& \cdot \sqrt{\left(q^{2} \rho^{2}+1\right)^{m+n-1} /\left(q^{2}+1\right)^{m+n}},
\end{aligned}
$$

При этом их значения в точках, расположенных на его боковой поверхности будут выражаться следующим образом:

$$
\begin{aligned}
& \sigma_{1}(R)=\sigma_{e}(R)[\cos \vartheta(R)+q \sin \vartheta(R)] / \sqrt{q^{2}+1}, \\
& \sigma_{3}(R)=\sigma_{e}(R)[q \cos \vartheta(R)-\sin \vartheta(R)] / \sqrt{q^{2}+1}
\end{aligned}
$$

Подставляя уравнения (12) соответственно в выражения (8) получим:

$$
\begin{gathered}
P=\frac{2 \pi R^{2} \sigma_{e}(R)}{\sqrt{\left(q^{2}+1\right)^{m+n}}} \cdot C[\vartheta(R), q, m, n], \\
M=\frac{2 \pi R^{3} \sigma_{e}(R)}{\sqrt{3\left(q^{2}+1\right)^{m+n}}} \cdot D[\vartheta(R), q, m, n],
\end{gathered}
$$

где выделены интегралы:

$$
\begin{aligned}
C[\vartheta(R), q, m, n]= \\
\quad=\int_{0}^{1} \rho\{\cos [\vartheta(R) \rho]+q \rho \sin [\vartheta(R) \rho]\} \sqrt{\left(q^{2} \rho^{2}+1\right)^{m+n-1}} \mathrm{~d} \rho
\end{aligned}
$$

и

$$
\begin{aligned}
D & {[\vartheta(R), q, m, n]=} \\
& =\int_{0}^{1} \rho^{2}\{q \rho \cos [\vartheta(R) \rho]-\sin [\vartheta(R) \rho]\} \sqrt{\left(q^{2} \rho^{2}+1\right)^{m+n-1}} \mathrm{~d} \rho .
\end{aligned}
$$

Систему уравнений (14) и (15) приводим к следующему виду:

$$
\frac{\sqrt{3} M}{P R}=\frac{D[\vartheta(R), q, m, n]}{C[\vartheta(R), q, m, n]} .
$$

Введем безразмерный параметр $Q=\sqrt{3} M / P R$ [19], где $R=R_{0} \sqrt{L_{0} / L(t)}$ - текущий радиус цилиндра. Если $q$ характеризует соотношение кинематических параметров процесса растяжения с кручением, то $Q$ - силовых параметров. Правая часть уравнения (18) представляет отношение интегралов $D[\vartheta(R), q, m, n]$ и $C[\vartheta(R), q, m, n]$, связывающих параметр деформирования $q$, постоянные материала в виде $(m+n)$ и угол сближения $\vartheta(R)$ между векторами напряжения и скоростей деформаций для точек на поверхности сплошного цилиндра. Эти интегралы в общем случае нельзя выразить при помощи элементарных функций, но для конкретных значений параметров $q, m$ и $n$ методом численного интегрирования определим значение $\vartheta(R)$. Подставляя полученное значение $\vartheta(R)$ в выражения (16) и (17) определим интегралы $D[\vartheta(R), q, m, n]$ и $C[\vartheta(R), q, m, n]$. Из уравнения (14) или (15) для точек цилиндра, расположенных на его боковой поверхности определим модуль вектора напряжений $\sigma_{e}(R)$, а с помощью выражений (13) его компоненты.

При пропорциональном деформировании когда $\vartheta(R)=0$, после интегрирования соответственно уравнений (14) и (15) получим формулы для определения интенсивности напряжений для материальной частицы, расположенной на боковой поверхности $\sigma_{e}(R)$, выраженные через осевое усилие $P$ и крутящий момент $M$ [19]:

$$
\begin{gathered}
\sigma_{e}(R)=\frac{P}{\pi R^{2}} \cdot \frac{(m+n+1) q^{2} \sqrt{\left(q^{2}+1\right)^{m+n}}}{2\left[\sqrt{\left(q^{2}+1\right)^{m+n+1}}-1\right]}, \\
\sigma_{e}(R)=\frac{\sqrt{3} M(m+n+3)}{2 \pi R^{3}} . \\
\cdot \frac{(m+n+1) q^{3} \sqrt{\left(q^{2}+1\right)^{m+n}}}{\sqrt{\left(q^{2}+1\right)^{m+n+1}}\left[(m+n+1) q^{2}-2\right]+2} .
\end{gathered}
$$

Из выражения (19), учитывая $q=\omega R / \sqrt{3} V$ при $\omega \rightarrow 0$ имеем формулу (4), а при $\mathrm{V} \rightarrow 0$ из (20) имеем формулу Филдса-Бэкофена (3), т.е. получили выражения для определения интенсивности напряжений при одноосном растяжении и при чистом кручении.

В работе [17] определены энергосиловые параметры процесса растяжения с кручением однородного цилиндрического стержня. В рамках модели нелинейно-вязкой жидкости $\sigma=K \xi^{m}$ были получены аналитические выражения для осевого усилия и крутящего момента (см. формулы (22) и (28) в [17]) выраженные через кинематические параметры (скорости растяжения и кручения) и константы материала $K$ и $m$. Эти выражения совпадают с выражениями (19) и (20) после подстановки $\sigma_{e}(R)=K \xi_{e}^{m}(R)$

Рассмотрим пример №2. Сплошной цилиндр нагружается по кинематической программе, состоящей из двух звеньев: первое звено - растяжение с осевой скоростью $V$ с одновременным кручением с угловой скоростью $\omega$; второе звено - одноосное растяжение со скоростью $V$. Во втором звене для одноосного растяжения $q=\omega R / \sqrt{3} V=0$ и уравнения (14) и (15) будут выглядеть следующим образом:

$$
\begin{gathered}
P=2 \pi R^{2} \sigma_{e}(R) \int_{0}^{1} \rho \cos [\vartheta(R) \rho] \mathrm{d} \rho, \\
M=\frac{2 \pi R^{3} \sigma_{e}(R)}{\sqrt{3}} \int_{0}^{1} \rho^{2}\{-\sin [\vartheta(R) \rho]\} \mathrm{d} \rho .
\end{gathered}
$$

После интегрирования этих уравнений получим:

$$
\begin{aligned}
& P=2 \pi R^{2} \sigma_{e}(R)\{\vartheta(R) \sin \vartheta(R)+\cos \vartheta(R)-1\} / \vartheta^{2}(R) \\
& M=2 \pi R^{3} \sigma_{e}(R) \cdot\left\{\left[\vartheta^{2}(R)-2\right] \cos \vartheta(R)-\right. \\
& \quad-2 \vartheta(R) \sin \vartheta(R)+2\} / \sqrt{3} \vartheta^{3}(R)
\end{aligned}
$$


Эту систему уравнений приводим к следующему виду:

$$
Q=\frac{\sqrt{3} M}{P R}=\frac{\left[\vartheta^{2}(R)-2\right] \cos \vartheta(R)-2 \vartheta(R) \sin \vartheta(R)+2}{\vartheta(R)\{\vartheta(R) \sin \vartheta(R)+\cos \vartheta(R)-1\}} .
$$

Связь между $9(R)$ и $Q=\sqrt{3} M / P R \quad$ представлена на Рис. S1 (дополнительный материал), где зависимость $\vartheta(R) \sim Q$ аппроксимирована полиномом второй степени для удобства обработки экспериментальных данных. Вектор напряжений при сближении поворачивается по часовой стрелке, угол $\vartheta(R)<0$. Подставляя полученное значение $\vartheta(R)$ в выражение (21) для точек образца, расположенных на его поверхности определим модуль вектора напряжений (интенсивность напряжений) и его компоненты:

$$
\begin{gathered}
\sigma_{e}(R)=P / 2 \pi R^{2} \cdot A, \quad \sigma_{1}(R)=\sigma_{e}(R) \cdot \cos \vartheta(R), \\
\sigma_{3}(R)=\sigma_{e}(R) \cdot\{-\sin \vartheta(R)\},
\end{gathered}
$$

где выделен множитель

$$
A=\vartheta^{2}(R) \cdot\{\vartheta(R) \sin \vartheta(R)+\cos \vartheta(R)-1\}^{-1},
$$

Из Рис. S2 (дополнительный материал) видно, что при исчерпании следа запаздывания, то есть при $\vartheta(R) \rightarrow 0 A=1$.

Рассмотрим пример № 3. Образец нагружается по кинематической программе, состоящей из двух звеньев: первое звено - растяжение с осевой скоростью $V$ с одновременным кручением с угловой скоростью $\omega$; второе звено - кручение с угловой скоростью $\omega$. Во втором звене для кручения $q=\omega R / \sqrt{3} V \rightarrow \infty$ и уравнения (14) и (15) будут выглядеть следующим образом:

$$
\begin{gathered}
P=2 \pi R^{2} \sigma_{e}(R) \int_{0}^{1} \rho^{m+n+1} \sin [\vartheta(R) \rho] \mathrm{d} \rho, \\
M=\frac{2 \pi R^{3} \sigma_{e}(R)}{\sqrt{3}} \int_{0}^{1} \rho^{m+n+2} \cos [\vartheta(R) \rho] \mathrm{d} \rho .
\end{gathered}
$$

Эту систему уравнений приводим к следующему виду:

$$
Q=\frac{\int_{0}^{1} \rho^{m+n+2} \cos [\vartheta(R) \rho] \mathrm{d} \rho}{\int_{0}^{1} \rho^{m+n+1} \sin [\vartheta(R) \rho] \mathrm{d} \rho} .
$$

Подставляя полученное (методом численного интегрирования) из (26) значение $9(R)$ в выражение (25) для точек образца, расположенных на его поверхности определим модуль вектора напряжений (интенсивность напряжений) и его компоненты:

$$
\begin{gathered}
\sigma_{e}(R)=\sqrt{3} M / 2 \pi R^{3} \cdot B, \quad \sigma_{1}(R)=\sigma_{e}(R) \cdot \sin \vartheta(R), \\
\sigma_{3}(R)=\sigma_{e}(R) \cdot \cos \vartheta(R),
\end{gathered}
$$

где выделен множитель

$$
B=\left\{\int_{0}^{1} \rho^{m+n+2} \cos [\vartheta(R) \rho] \mathrm{d} \rho\right\}^{-1},
$$

Изменение множителя $B$ в зависимости от угла сближения $\vartheta(R)$ и коэффициента скоростной чувствительности материала $m$ показано на Рис. S3 (дополнительный материал). Видно, что при исчерпании следа запаздывания, т.е. при $\vartheta(R) \rightarrow 0$ ( $B=3$ для идеально пластической среды $(m=0)$ и $B=4$ для ньютоновской вязкой жидкости $(m=1))$ имеем формулу Филдса-Бэкофена (3).
Практически обработка зарегистрированных в цифровом виде данных испытания производится с использованием электронной таблицы, например Microsoft Excel и заключается в следующем. После вычисления текущей длины $L(t)=L_{0}+\Delta L(t)$, текущего радиуса $R(t)=R_{0} \sqrt{L_{0} / L(t)}$, кинематического параметра $q(t)=\omega R(t) / \sqrt{3} V(t)$ и силового параметра $Q(t)=\sqrt{3} M(t) / P(t) R(t)$ используя соотношение (18) определяем $9(R)(t)$, из соотношений (14) и $(15) \sigma_{e}(t)$ и затем по формулам $(13) \sigma_{1}(R)(t)$ и $\sigma_{3}(R)(t)$.

Предлагаемая методика была использована при обработке экспериментальных данных, полученных при испытании титанового сплава Ti-6Al-4V при непропорциональном деформировании в условиях сверхпластичности. Объектом испытания был сплошной цилиндрический образец диаметром рабочей части 10 мм и длиной рабочей части 35 мм. В средней части образца, где деформация практически однородна, двумя рисками ограничивали расчетную длину. Для учета неравномерности удлинения по длине образца определяли приведенную начальную длину образца по формуле: $L_{0}=\Delta L_{k} l_{0} /\left(l_{k}-l_{0}\right)$, где $l_{0}, l_{k}-$ начальная и конечная расчетная длина (расстояние между рисками); $\Delta L_{k}-$ абсолютное удлинение образца после окончания испытания (расстояние между головками образца).

Программа испытания показана на Рис. S4 (дополнительный материал). Она состояла из трех звеньев: первое звено- одноосное растяжение; второе звено (с 140 по 230 сек.) - растяжение с одновременным кручением; третье звено (с 230 по 280 сек.) - одноосное растяжение. Скорость растяжения во всех звеньях была одинаковой и составляла $V=0.0215$ мм/с, скорость кручения во втором звене составила $\omega=0.026 \mathrm{paд} / \mathrm{c}$. Температура испытания $-900^{\circ} \mathrm{C}$. Коэффициенты скоростного и деформационного упрочнения материала, определенные ранее, составили соответственно $m=0.32$ и $n=0$. В процессе испытания каждую секунду регистрировали следующие параметры: текущее время $t$, абсолютное удлинение $\Delta L(t)$, угол закручивания $\varphi(t)$, осевую силу $P(t)$ и крутящий момент $M(t)$. Количество записей в минуту -60 . Полученные данные обрабатывали с помощью электронной таблицы "Microsoft Excel". Текущую длину образца $L(t)$ вычисляли по формуле: $L(t)=L_{0}+\Delta L(t)$, а текущий радиус $R(t)$ вычисляли из условия объемной несжимаемости материала по формуле: $R(t)=R_{0} \sqrt{L_{0} / L(t)}$.

Первичные диаграммы изменения осевого усилия $P(t)$ и крутящего момента $M(t)$ в зависимости от времени $t$ показаны на Рис. S5 (дополнительный материал). Видно, что во втором звене кручение приводит не только к появлению крутящего момента, но и к уменьшению осевого усилия. После выключения кручения наблюдается увеличение осевого усилия. За счет нежесткости (податливости) системы «образец - испытательная машина» изменение осевого усилия и крутящего момента приводит к изменению заданной в соответствии c Рис. S4 (дополнительный материал) скорости деформирования образца. Для материалов, чувствительных к скорости деформации это приводит к искажению диаграммы деформирования [20]. В данных условиях испытания податливость на осевое растяжение соста- 
вила $1.7 \times 10^{-4} \mathrm{Mm} / \mathrm{H}$, а на кручение $-8 \times 10^{-6}$ рад $/ \mathrm{H} \times$ мм Изменение скоростей осевого деформирования и кручения образца с учетом податливости системы «образец - испытательная машина» показано на Рис. S6 (дополнительный материал). Их значения определяли по формулам: $V(t)=0.0215-1.7 \cdot 10^{-4} \cdot \mathrm{d} P / \mathrm{d} t(\mathrm{Mм} / \mathrm{c})$ и $\omega(t)=0.026-8 \cdot 10^{-6} \cdot \mathrm{d} M / \mathrm{d} t$ (рад/сек). Таким образом, после включения и выключения кручения действительная траектория деформации выходит на прямолинейный участок с некоторым запаздыванием, то есть вместо излома траектории имеем траекторию переменной кривизны. Поэтому, для каждого значения $V(t)$ и $\omega(t)$ в переходных участках уточняли кинематический параметр $q(t)=\omega R(t) / \sqrt{3} V(t)$.

В первом звене напряжения определяли по формуле (4): $\sigma_{e}(t)=\sigma_{1}(t)=P(t) / \pi R^{2}(t)$. Обработку диаграмм $P(t)$ и $M(t)$ во втором и третьем звене проводили в соответствии с вышеизложенной методикой с учетом изменения $V(t)$ и $\omega(t)$ в переходных участках. На Рис. S7 (дополнительный материал) приведено изменение угла сближения на поверхности образца $\vartheta(R)$ по длине второго звена $\Delta S$. Зависимость модуля вектора напряжения (интенсивности напряжений) и его компонент на поверхности сплошного цилиндрического образца от времени представлена на Рис. S8 (дополнительный материал).

Приведем также данные испытания при температуре $900^{\circ} \mathrm{C}$ тонкостенного трубчатого образца наружным диаметром 14 мм, толщиной стенки 1 мм и длиной рабочей части 50 мм, изготовленного из титанового сплава Ti-6Al-4V. Программа испытания также задавалась по деформациям и состояла из трех этапов. Первый этап - осевое растяжение со скоростью 0.3 мм/с; второй этап - осевое растяжение со скоростью $0.17 \mathrm{Mм} / \mathrm{c}$ и третий этап - осевое растяжение со скоростью 0.17 мм/с с одновременным кручением со скоростью 0.09 рад/с. На третьем этапе трубчатый образец потерял устойчивость. Напряжения определяли по следующим формулам:

$$
\sigma_{1}=P / 2 \pi R \delta, \quad \sigma_{3}=M / 2 \pi R^{2} \delta, \quad \sigma_{e}=\sqrt{\sigma_{1}^{2}+\sigma_{3}^{2}},
$$

где $P$ и $M-$ соответственно текущее осевое усилие и текущий крутящий момент; $R-$ средний радиус образца; $\delta$ - толщина стенки образца.

Значения модуля вектора напряжения (интенсивности напряжений) $\sigma_{e}$ и его компонент $\sigma_{1}, \sigma_{3}$ при деформировании трубчатого образца из титанового сплава Ti-6Al-4V приведены на Рис. S9 (дополнительный материал). Буквой «A» отмечен момент потери устойчивости образца. На Рис. S10 (дополнительный материал) показано изменение угла сближения $\vartheta$ по длине третьего этапа после излома траектории деформации.

Изменение угла сближения после излома траектории деформации определяет ориентацию вектора напряжений относительно траектории деформации. Как для трубчатого (Рис. S10, дополнительный материал), так и для сплошного (Рис. S7, дополнительный материал) образца характерно, что по исчерпанию деформации $\sim 1.5 \%$, то есть следа запаздывания, угол между векторами напряжения и скоростей деформаций стано- вится достаточно малым и вектор напряжений практически ложится на траекторию деформаций.

Что касается напряжений, то после излома траектории деформаций на характер их изменения определенное влияние оказывает нежесткость системы «образец - испытательная машина». Изменение скорости деформации за счет нежесткости приводит к изменениям на диаграмме деформирования материала, чувствительного к скорости деформации. Эти особенности переходных участков характерны как для трубчатого, так и для сплошного образцов.

Представленная методика является обобщением экспериментальной методики построения диаграммы сдвига по данным испытаний на кручение материала, чувствительного к скорости и степени деформации, основанной на формуле Филдса-Бэкофена. Она позволяет найти значение вектора напряжений и его ориентацию по отношению к вектору скорости деформации из экспериментальных зависимостей $P(t)$ и $M(t)$, которые регистрируются в опытах на непропорциональное деформирование (сложное нагружение) по ломаной траектории с использованием сплошных цилиндрических образцов.

Благодарности/Acknowledgements. Работа выполнена в рамках государственного задания ИПСМ РАН./The present work was supported by the state assignment of IMSP RAS.

Дополнительныйматериал/SupplementaryMaterial. Электронная версия статьи содержит дополнительный материал (рисунки), доступный безвозмездно на сайте журнала (www.lettersonmaterials.com)./The online version of this paper contains supplementary material (figures) available free of charge at the journal's Web site (www.lettersonmaterials.com).

\section{Литература/Reference}

1. A.A. Ilyushin. Plasticity. Fundamentals of general mathematical theory. Moscow, The USSR AS (1963) 272 p. (in Russian) [А. А. Ильюшин. Пластичность. Основы общей математической теории. Москва, АН CCCP (1963) 272 c.]

2. V.G. Zubchaninov. Foundations of theory of elasticity and plasticity. Moscow, High School (1999) 368 p. (in Russian) [В. Г. Зубчанинов. Основы теории упругости и пластичности. Москва, Высш. Школа (1999) 368 с.]

3. V.S. Bondar, V.V. Danshin. Plasticity. Proportional and non-proportional loading. Moscow, FIZMATLIT (2008) 176 p. (in Russian) [В. С. Бондарь, В. В. Даншин. Пластичность. Пропорциональные и непропорциональные нагружения. Москва, ФИЗМАТЛИТ (2008) 176 c.]

4. B. D. Annin, V. M. Jigalkin. Behavior of materials under conditions of complex loading. Novosibirsk, SO RAS (1999) 342 р. (in Russian) [Б. Д. Аннин, В. М. Жигалкин. Поведение материалов в условиях сложного нагружения. Новосибирск, СО РАН (1999) 342 с.] 
5. R.A. Vasin. In: Plastichnost i razrushenie tverdyih tel. Moscow, Nauka (1988) Ob eksperimental'nom issledovanii... P. 40 - 57. (in Russian) [Р. А. Васин. В кн.: Пластичн. и разрушение тверд. тел. Москва, Наука (1988). Об экспериментальном исследовании функционалов пластичности в теории упругопла-стических процессов. С. 40 - 57.]

6. R. A. Vasin, A. N. Nikitochkin, P. M. Ogibalov. Mechanics of Polymers. 4, 739 (1973). (in Russian) [Р. А. Васин, А.Н. Никифоров, П. М. Огибалов. Механика полимеров. 4,739 (1973).]

7. R. A. Vasin, A. N. Nikitochkin, P. M. Ogibalov. Mechanics of Polymers. 2, 224 (1975). (in Russian) [Р. А. Васин, А.Н. Никифоров, П. М. Огибалов. Механика полимеров. 2, 224 (1975).]

8. A.V. Muravlev. In: Proceedings of VIII International scientific workshop "Problemy prochnosti, plastichnosti i ustoichivosti $\mathrm{v}$ mehanike deformiruemogo tverdogo tela". Tver (2015) Р. 25. (in Russian) [А. В. Муравлёв. Материалы VIII Международного научного симпозиума "Проблемы прочности, пластичности и устойчивости в механике деформируемого твердого тела". Тверь (2015) C. 25.]

9. K.Zhang, M. K. Khraisheh, A. E. Bayoumi, C.H. Hamilton, H. M. Zbib. Proceeding ICSAM-94. (1994) P. 583 - 588.

10. Y. Ohashi, M. Tokuda. J. Mech. Phys. Solids 21, 241 (1973).

11. M. Tokuda, Y. Inagaki, H. Yoshida. JSME International Journal. A. 37(2), 117 (1993).
12. V.G. Zubchaninov, N.L. Ohlopkov. Strench of Materials. 4, 19 (1996). (in Russian) [В.Г. Зубчанинов, Н. Л. Охлопков. Проблемы прочности. 4, 19 (1996).]

13. V.G. Zubchaninov, V.I. Gultiaev, A.A. Alekseev, V.V. Garanikov, S.L. Subbotin. Mater. Phys. and Mech. 32, 305 (2017).

14. R. A. Vasin, A.A. Ilyushin, P.A. Mossakovski. Solid Mechanics. 2, 177 (1994). (in Russian) [Р.А. Васин, А. А. Ильюшин, П.А. Моссаковский. Механика твердого тела. 2, 177 (1994).]

15. A. V. Muravlev. In: Vestnik MGU, ser. Mat. Mech. 5, 74 (1996). (in Russian) [А. В. Муравлев. Вест. МГУ, сер. Мат. Mex. 5, 74 (1996).]

16. H. J. McQueen, W. Blum and Q.Zhu. Superplasticity in Advanced Materials, ICSAM-94, Moscow, T. G. Langdon (ed.), Trans. Tech. Pub., Zurich, Switzerland, 1994, P. $193-200$.

17. F. U. Enikeev. Metals. 4, 66 (1999). (in Russian) [Ф. У. Еникеев. Металлы. 4, 66 (1999).]

18. R.A. Vasin, V.K. Berdin, R.M. Kashaev. Strength of Materials. 6, 509 (2001). [Р.А. Васин, В.К. Бердин, P.М. Кашаев. Проблемы прочности. 6, 5 (2001).]

19. V.K. Berdin, R. M. Kashaev. Strength of Materials. 1, 15 (2001). [В.К. Бердин, Р.М. Кашаев. Проблемы прочности. 1, 28 (2001).]

20. R. A. Vasin, F. U. Enikeev. Introduction to superplasticity mechanics. Part 1. Ufa, Gilem (1998) 280 p. (in Russian) [Р.А. Васин, Ф.У. Еникеев. Введение в механику сверхпластичности. Ч. 1. Уфа, Гилем (1998) 280 с.] 